\title{
Leriche Syndrome in a post-CABG patient
}

\author{
Cristina Luca ${ }^{*}, 1,2$, Alin Ciubotaru1, Carmen Leferman", Radu Anghel ${ }^{1}$, Elena Deju², \\ Grigore Tinică ${ }^{1,2}$
}

1 "Grigore T. Popa" University of Medicine and Pharmacy lași, ${ }^{2}$ Institute for Cardiovascular Diseases

"Prof. Dr. George I. M. Georgescu", lași, Romania

\begin{abstract}
We report the case of a 48 years-old man diagnosed with Leriche syndrome. The patient had no angina, rare palpitations and intermittent claudication with a walking perimeter about 500 meters. The patient had numerous cardiovascular risk factors and he underwent a percutaneous transluminal coronary angioplasty (PTCA) on right coronary artery (RCA) and on left anterior descending (LAD) artery thirteen years ago. In-stent restenosis was found two years after PTCA thus, a primary isolated coronary artery bypass graft (CABG) surgery was performed using an in situ left internal mammary artery (LIMA) graft to LAD and a radial artery graft on RCA. Eleven years post-CABG surgery, a coronary CT angiography examination revealed that the radial graft on RCA and the native right coronary artery were occluded, but in situ LIMA graft was permeable. The CT angiography of the abdominal aorta and lower limbs showed that the aortic bifurcation and common iliac arteries were occluded.
\end{abstract}

Keywords: Leriche syndrome, bypass graft, CT angiography, coronary artery bypass graft

\section{Introduction}

Aorto-iliac occlusive disease was described by Robert Graham in 1814. Leriche syndrome was named after a famous French surgeon, René Leriche, who successfully operated and reported "his" syndrome in 1923, on a 29 years-old truck driver [1]. Leriche syndrome refers to occlusion of the abdominal aorta distal to the renal arteries, aortic bifurcation and the common iliac arteries being usually involved. Occlusive disease involving the aorto-iliac segment develops especially in patients with risk factors or conditions that increase clotting of blood: hyperlipidemia, hypertension, diabetes mellitus, smoking and usually affects younger males ages 30-40.

Received: November 2014; Accepted after review: March 2015; Published: March 2015

*Corresponding author: Assist. Prof. Cristina Luca, $\mathrm{MD}, \mathrm{PhD}$, "Grigore T. Popa" University of Medicine and Pharmacy lași; "Prof. Dr. George I.M. Georgescu" Institute for Cardiovascular Diseases lași, Romania.

E-mail: cristinavartic@yahoo.com
The vessels above and below may be relatively normal. It is often associated with chronic renal failure and coronary artery disease [2].

Chronic lower extremity ischemia induces a triad of symptoms: intermittent claudication in the legs or buttocks, erectile dysfunction and absent or diminished femoral pulses [3]. If aortic stenosis develops slowly, collateral vascular circulation can develop.

\section{Case presentation}

We present the case of a 48 years-old patient who underwent a primary isolated coronary artery bypass surgery at age of 37 . In June 2003 he was diagnosed with acute anterior myocardial infarction non-Q. The coronarograhpy revealed an in-stent restenosis of previously percutaneous transluminal coronary angioplasty (PTCA) on right coronary artery (RCA) in 2001 and on left anterior descending (LAD) artery in 2002. A primary isolated coronary artery bypass graft 
(CABG) surgery was performed at Institute for Cardiovascular Diseases "Prof. Dr. George I. M. Georgescu" lași. Surgical myocardial revascularization was done by using a radial artery graft on RCA and an in situ left internal mammary artery (LIMA) graft on LAD. The postoperative evolution was favorable, with the resolution of angina and wound healing per primam.

We performed the grafts patency evaluation in August 2014 at Institute for Cardiovascular Diseases "Prof. Dr. George I. M. Georgescu" lași on a second-generation 128-slice DSCT scanner (Somatom Definition Flash; Siemens Healthcare, Forchheim, Germany). Scanning parameters were $120 \mathrm{kV}$ tube voltage, tube current modulation with Care Dose 4D, $128 \times 0.6 \mathrm{~mm}$ collimation. Peak arterial enhancement time was determined by the bolus tracking method. The contrast media for coronary CT angiography was injected using a power injector at 6 $\mathrm{ml} / \mathrm{sec}$, followed by $50 \mathrm{ml}$ saline flush. Cardiac images were reconstructed with a slice thickness of $0.75 \mathrm{~mm}$, using a soft-tissue convolution kernel (B26f) and a sharp-tissue convolution kernel (B46) for vessel wall calcifications, to compensate blooming artefacts.

Coronary CT angiography examination showed that the radial graft on RCA and the native right coronary artery were occluded. There was a in situ permeable LIMA graft that supply the LAD, and arterial anastomoses between the distal LAD and the collateral rami of RCA (retroventricular ramus for right ventricle and right posterolateral system) (Figure 1 and Figure 2).
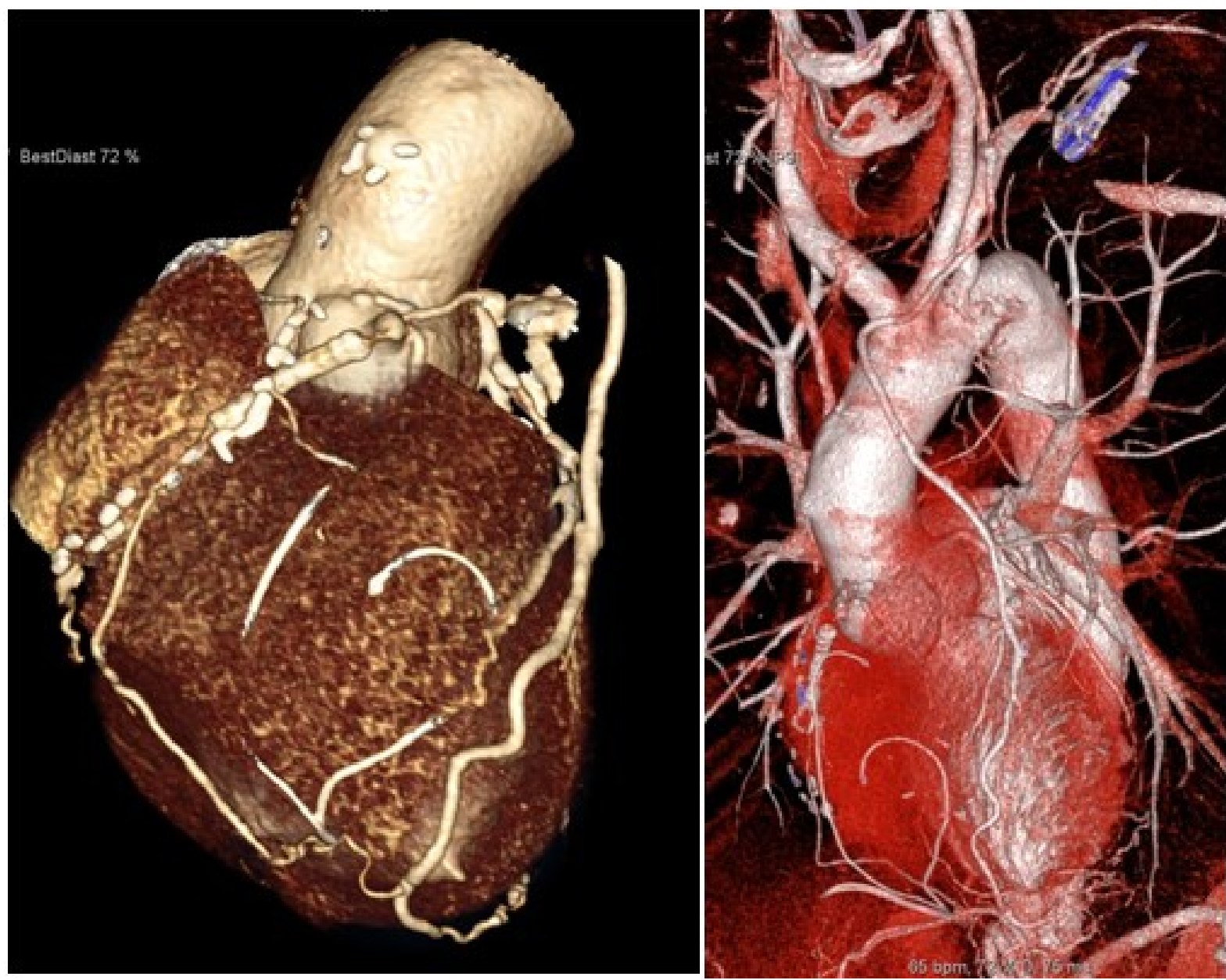

Fig. 1. Three dimensional reconstruction with volume rendering techniques (VRT) - occluded radial graft on right coronary artery (RCA), occluded stented native RCA; permeable in situ LIMA graft on LAD. 


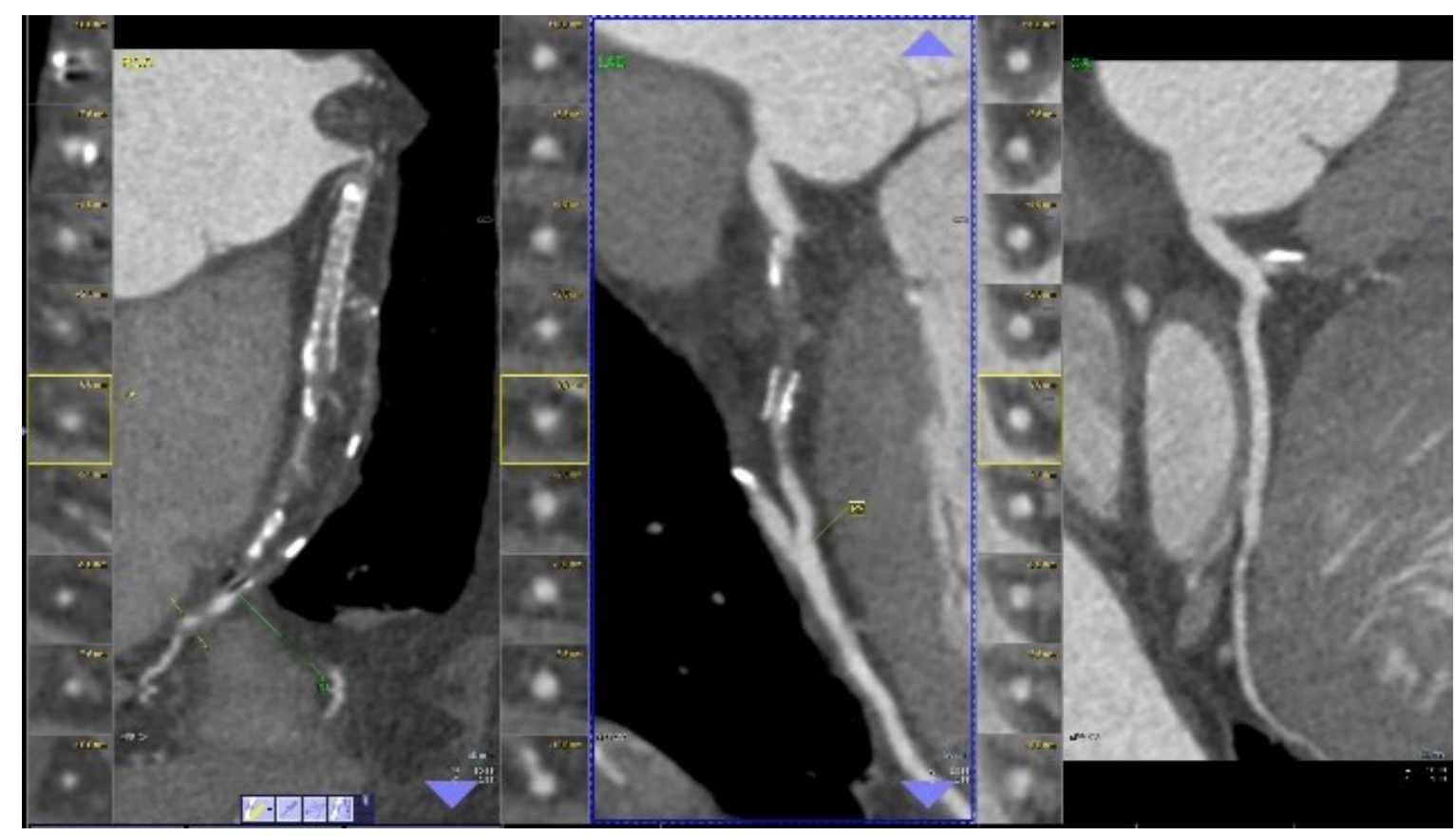

Fig. 2. Curved planar reformation (CPR) images of coronary CT angiography - occluded stented native RCA, occluded radial graft on RCA, occluded stented LAD proximal to LIMA graft anastomose, permeable LCx artery

At the level of the last axial cross-section of the initial cardiac acquisition, just below the emerging of superior mesenteric artery (SMA), we saw an important parietal thrombosis of the abdominal aorta. At that level, aorta was about
$3 \mathrm{~cm}$ diameter but its permeable lumen was $1.7 / 1.3 \mathrm{~cm}$. An important arterial collateral network of right internal thoracic artery was developed into the right rectus sheath (Figure $3)$.

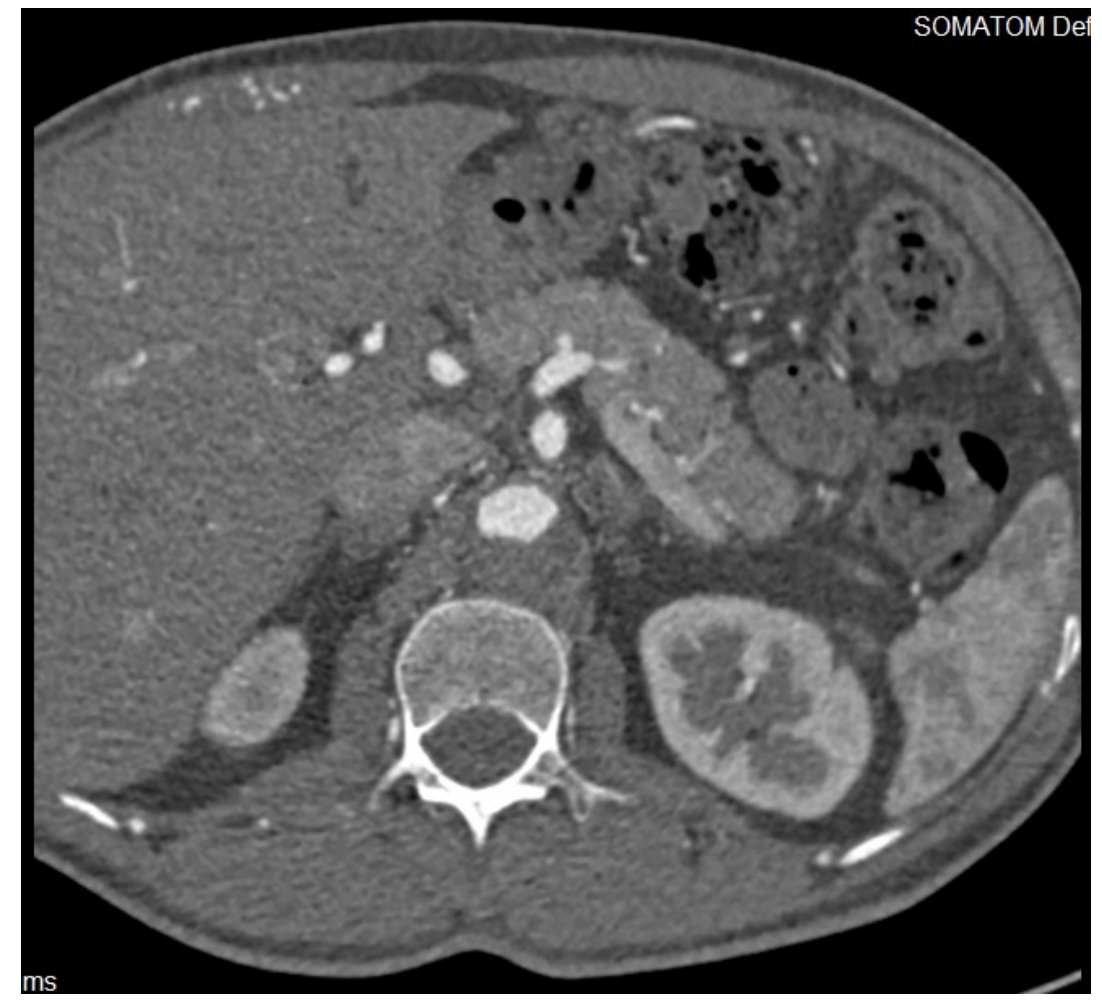

Fig. 3. The last axial cross-section image of coronary CT angiography: important parietal thrombosis of abdominal aorta; important arterial network into right abdominal rectus sheath, neither into the left one 
At the clinical examination, the patient has no angina, rare palpitations and intermittent claudication with a walking perimeter about 500 meters. He is a smoker of 10 cigarettes per day and he also has polydipsia. Its blood pressure was $160 / 80 \mathrm{mmHg}$, heart rate $90 /$ minute, without cardiac decompensation signs, without cardiac murmurs. The pulse was absent at left superficial femoral artery and bilaterally at the posterior tibial arteries.

According to coronary CT angiography result and clinical signs and symptoms we decided to examine the abdominal aorta and inferior limbs using CT angiography. The angiography showed that the patient abdominal aorta was occluded inferior to the renal arteries. At the emerging of celiac trunk, abdominal aorta presented circumferential parietal thrombosis, about $7.4 \mathrm{~mm}$ thickness. At the SMA emerging the parietal thrombosis was $1.05 \mathrm{~cm}$ thickness. There was no stenosis of celiac trunk or SMA emerging (Figure 4). The parietal thrombosis circumscribes the two ostia of renal arteries causing critic stenoses of $75-80 \%$ on the left side and $85-90 \%$ on the right side (Figure 5).
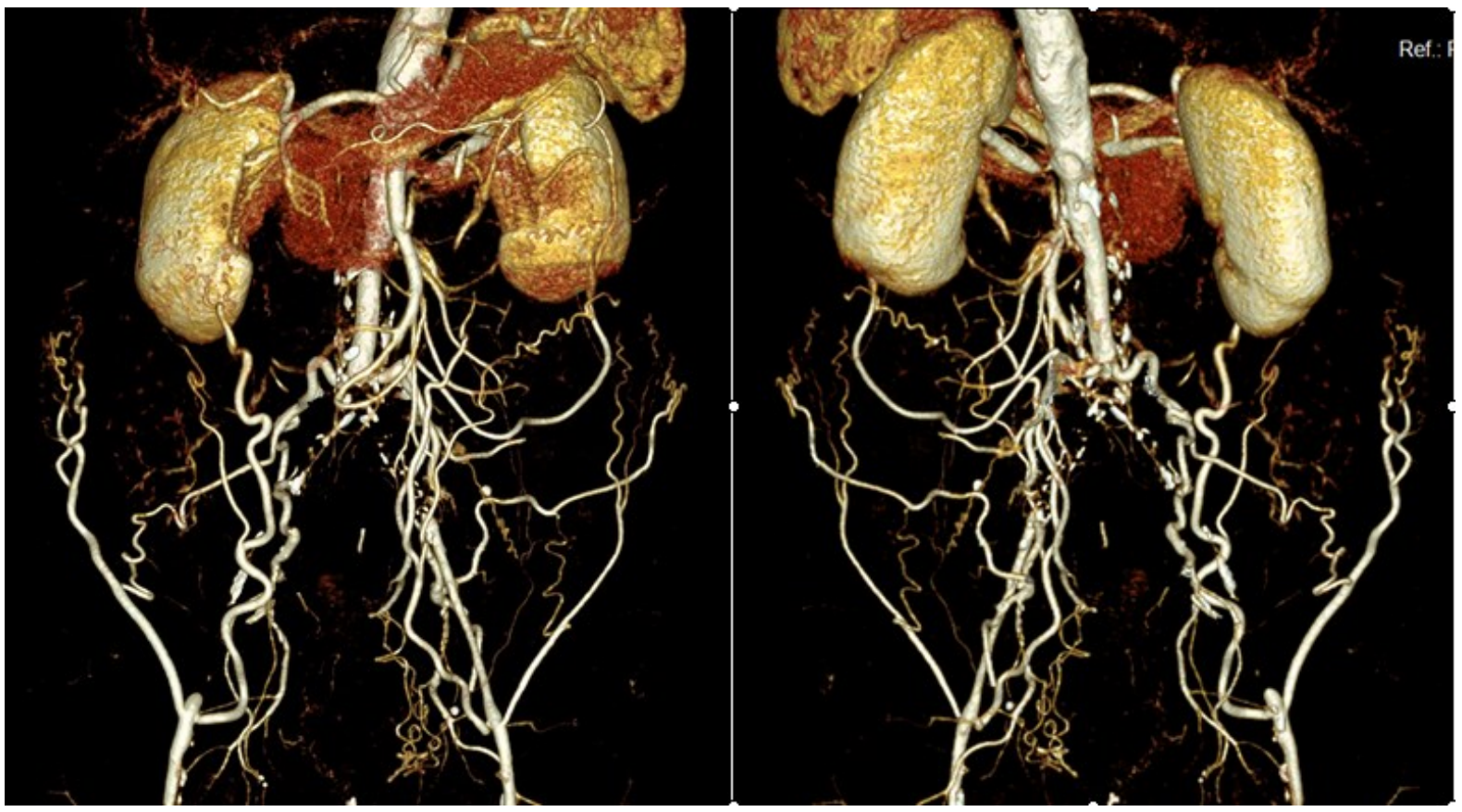

Fig. 4. Three dimensional reconstruction with volume rendering techniques (VRT) shows the occluded aorta, the occluded common iliac arteries and the arterial anastomoses that supply the inferior limbs (anterior and posterior views)

At the emerging of renal arteries, parietal thrombosis was eccentric, about $1.54 \mathrm{~cm}$ thickness, leaving an anterior permeable aortic lumen of $1.7 / 1.27 \mathrm{~cm}$. Distally to the renal arteries, the aortic permeable lumen becomes increasingly tapered, parietal thrombosis was concentric and, at the level of the $4^{\text {th }}$ lumbar vertebra, the abdominal aorta becomes occluded. The permeable aortic distal lumen gives rise to two well opacified voluminous lumbar collateral arteries, establishing anastomoses with internal iliac arteries.
The aortic bifurcation and common iliac arteries were occluded.

On the right side, the right internal iliac artery (RIIA) was supplied by the right lumbar collateral artery, but the right external iliac artery (REIA) was occluded. Right common femoral artery was supplied by right deep inferior epigastric artery and right deep iliac circumflex artery. Right superficial femoral, deep femoral and popliteal arteries were permeable, without atheromatosis or stenosis. The arteries of the right leg were normal. 
On the left side, the left internal iliac artery (LIIA) was supplied by the left lumbar collateral artery. Only the left common iliac artery bifurcation was opacified. The left external iliac artery (LEIA) was supplied by collateral circulation. Left deep inferior epigastric and left deep iliac circumflex arteries supplied the left common femoral artery. Left superficial femoral, deep femoral and popliteal arteries were permeable, without stenosis. The arteries of the left leg were normal. There was an important pelvic and abdominal parietal collateral circulation.

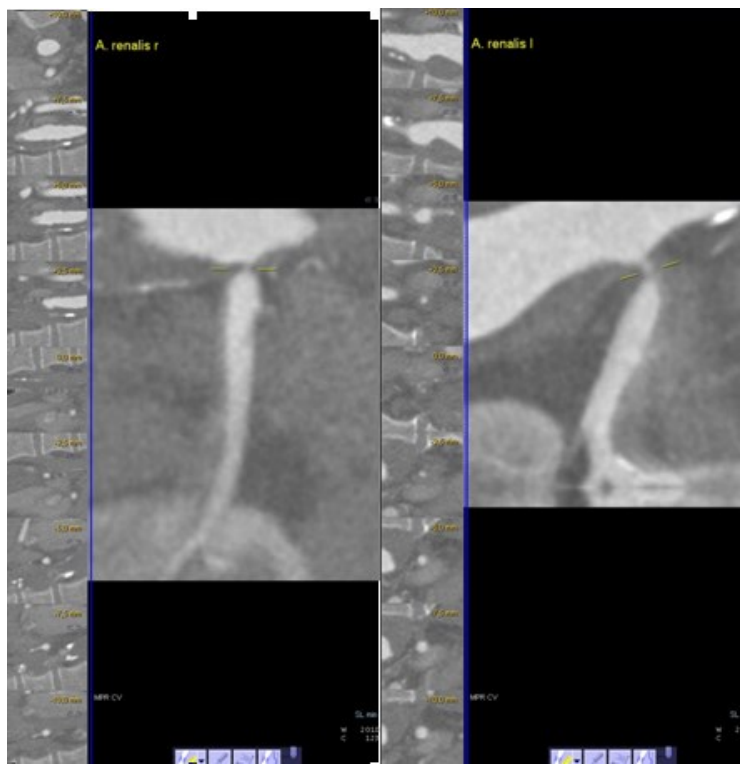

Fig. 5. Curved planar reformation (CPR) images of CT angiography - stenotic renal arteries

\section{Discussion}

The imaging diagnosis in this case was Leriche syndrome.

The embryologic development of the abdominal and lower extremity arteries allows collateralization flow [4]. That is why in pacients affected by aorto-iliac occlusive disease, an important parietal and visceral arterial network develops. The role of this network is to supply all structures of the lower extremity by means of collateral channels [5]. There are four common collateral circulatory pathways: a. the superior mesenteric and inferior mesenteric arteries can supply each other in a bidirectional manner through Riolan' $s$ arterial arch [6]; b. the inferior mesenteric artery is anastomosed with the internal iliac artery via the hemorrhoidal plexus. Visceralvisceral collateral pathways that exist between celiac trunk and its divisions, SMA, IMA, and superior rectal artery are due to their common origin. The extension of atherosclerotic disease will determine these pathways to be used; c. The Winslow pathway involves internal thoracic (mammary) arteries that supply the external iliac arterial flow via the anastomoses between superior epigastric and inferior epigastric arteries into the abdominal rectus sheath [7]; $d$. The intercostal, subcostal and lumbar arteries supply the external iliac arteries through the superficial and deep iliac circumflex arteries or through the gluteal arteries (via internal iliac arteries).

The systemic-systemic collateral pathways include the lumbar, intercostal, deep circumflex iliac, internal thoracic, inferior epigastric and obturator arteries. These pathways derive from the embryologic segments of the dorsal aorta. Distal abdominal aorta occlusion tends to collateralize through systemic-systemic pathway $[8,9,10]$. This case has a particular condition by using the left internal mammary artery to revascularize the LAD artery. There was only right side anastomosis between right internal thoracic artery and right inferior epigastric artery. In a patient with aortoiliac occlusive disease the lower extremity arterial flow is dependent on these arteries and such a condition can cause lower extremity claudication [11]. Moreover, the course of this artery may put it at risk to be cut during abdominal surgery.

The arterial supply of the lower limbs of our patient was not a critical one due to development of these particular collateral pathways. In this case, the parietal arterial anastomoses provide blood for the common femoral arteries via the inferior epigastric and deep iliac circumflex arteries. Moreover, the internal iliac arteries were supplied by two voluminous lumbar arteries raised from the end of the aortic permeable lumen. These findings determine the surgeon to temporize the surgical intervention for Leriche syndrome. The main priority in this case was represented by the critical stenoses of renal arteries that were managed through percutaneous transluminal angioplasty. 
Another particularity in this case is represented by particular anastomoses between left and right coronary arteries. On the right side, also the radial arterial graft and the native right coronary artery are occluded. The left coronary artery succeed to supply the right coronary territory although in this case the coronary system was right dominated. Visible on CTA were the anastomoses between the distal LAD and the rami of the right coronary artery (retroventricular ramus for right ventricle and posterolateral system).

\section{Conclusion}

The left internal mammary artery is the best choice in cardiac surgery to revascularize the left anterior descending artery. In the case

\section{References}

1. Lee WJ, Cheng YZ, Lin HJ. Leriche syndrome. Int J Emerg Med 2008; 1(3): 223.

2. Keller K, Beule J, Oliver Balzer JO, et al. A 56 year-old man with co-prevalence of Leriche syndrome and dilated cardiomyopathy: case report and review. Wien Klin Wochenschr 2014; 126(56):163-168.

3. Frederick M, Newman J, Kohlwes J. Leriche Syndrome. J Gen Intern Med 2010; 25(10):1102-1104.

4. William PL. Gray's Anatomy. 38th Ed. London: Churchill Livingstone, 1995.

5. Ferrer MC, Calvo I, Sánchez-Rubio J, et al. The importance of investigating the internal thoracic artery before coronary artery surgery in Leriche's syndrome. Rev Esp Cardiol 2007; 60(11):1198-1201.

6. Lange JE, Komen N, Akkerman G, et al. Riolan's arch: confusing, misnomer, and obsolete. A literature survey of the connections between the superior and of patients with Leriche syndrome, acute ischemia of the lower limbs has been reported when the IMA was used in bypass grafting. In these patients, the IMA can be the main source of collateral circulation to the femoral arteries and its use in myocardial revascularization is associated with acute lower limbs ischemia. The percentage of flow to the lower limbs supplied by the mammary arteries depends on the location of the abdominal aorta obstruction, and it is greater when the obstruction is more proximal. Our patient did not have abdominal aorta flow immediately after the origin of the renal arteries, and despite using the left IMA in coronary revascularization, his quality of life was not affected in a critical way. The ideal treatment for these patients is still uncertain, because the experience is limited in this area.

inferior mesenteric arteries. Am J Surg 2007; 193(6):742-748.

7. Covey AM, Pua B, Aguado A, Madoff DC. Interventional radiology cases. Oxford: Oxford University Press, 2014.

8. Hardman R, Lopera J, Cardan R, Trimmer $\mathrm{C}$, Josephs S. Common and rare collateral pathways in aortoiliac occlusive disease: a pictorial essay. Am J Roentgenol 2011; 197(3):W519-W524.

9. Yurdakul M, Tola M, Özdemir E, Bayazit $\mathrm{M}$, Cumhur $\mathrm{T}$. Internal thoracic arteryinferior epigastric artery as a collateral pathway in aortoiliac occlusive disease. $J$ Vasc Surg 2006; 43(4):707-713.

10. Green CS, Helmy MA. Novel, congenital iliac arterial anatomy: Absent common iliac arteries and left internal iliac artery. Radiology Case Reports 2014; 9(3):978.

11. Ferrer MC, Calvo I, Sánchez-Rubio J, et al. The importance of investigating the internal thoracic artery before coronary artery surgery in Leriche's Syndrome. Rev Esp Cardiol 2007; 60(11):1198-1201. 